\title{
Experiential Teaching-learning Tools: Critical Study of Representational Media and Immersion in Architecture
}

\author{
Carolina Carrasco-Walburg ${ }^{1}$, Eduardo Valenzuela-Astudillo ${ }^{1}$, Sandro Maino- \\ Ansaldo $^{1}$, Matías Correa-Díaz ${ }^{1}$, Diego Zapata-Torres ${ }^{1}$ \\ ${ }^{1}$ Universidad Técnica Federico Santa María, Chile \\ carolina.carrasco@usm.cl \\ eduardo.valenzuelaa@usm.cl \\ sandro.maino@usm.cl \\ matias.correa.14@sansano.usm.cl \\ diego.zapatat@sansano.usm.cl
}

\begin{abstract}
.
The use of Virtual Reality (VR) in teaching-learning process of design, theory and history of architecture has increased in terms of virtual tours. A preliminary study of techniques and capabilities of Immersive Virtual Reality (IVR) systems allowed us to establish that the immersive and interactive virtual experience facilitates the perception and enhancement of spatial qualities. In addition, it facilitates analysis since it promotes observation and the development of spatial thinking. However, the use of this medium as a tool for analysis is less frequent. Therefore, in this research we comparatively evaluate the impact that VR has on such a task. We developed an analysis instrument using experiential learning cycles that was tested with students in control and experimental groups. As a result, we found that the experience of inhabiting facilitates integration of fundamental concepts, allowing empirical evaluation of architecture and streamlining communication in the classroom as an active learning strategy.
\end{abstract}

Keywords: Virtual Reality, Architecture, Spatial Perception, Experiential Learning, Teaching-Learning Process. 


\section{Introducción}

\subsection{Antecedentes y Estado del Arte}

La realidad virtual (RV) es una herramienta idónea para potenciar la estrategia de enseñanza-aprendizaje del diseño arquitectónico, que ya se explora a nivel mundial (Radianti et.al, 2020). También existe evidencia de los variados usos aplicados a la enseñanza de la historia de la arquitectura (Ge, 2019; Kowalski, Samól, Szczepański, J.\& Dłubakowski, 2020; Sooai et.al, 2019) y al ámbito del patrimonio donde se viene discutiendo su aporte en la exploración científica y valorización patrimonial (Gutiérrez, Hernández, 2003; Hu, Ng, \& Lee, 2019).

La RV no viene a reemplazar los campos tradicionales de la arquitectura, sino a complementarlos ofreciendo nuevas posibilidades. Ayuda a visualizar y controlar información compleja -espacialidad, tridimensionalidad- que, de entrada, nuestro cerebro no puede procesar (Gutiérrez, Hernández, 2003). En buena medida, cumple un rol fundamental en la formulación de nuevas estrategias docentes basadas en la integración de tecnologías de la información y comunicación (TIC) (Delgado, Fonseca, 2017). Sin embargo, en los últimos trabajos observados se identifica una baja presencia o declaración explícita de teorías de aprendizaje implementadas para el diseño de las experiencias, así como, una escasa exploración en los contenidos del aprendizaje (Radianti et.al, 2020).

Este medio logra generar sensación de presencia en el medio digital (virtual), siendo esta una de sus principales características, a la vez que permite desarrollar una amplia gama de interacciones, diversas en forma y efecto (Levis, 1997). Se ha demostrado que como tecnología de visualización la RV logra facilitar la representación de arquitectura (Delgado, Fonseca, 2017). También que la experiencia inmersiva promueve la percepción espacial gracias a la relación cuerpo-espacio facilitando la comprensión de las dimensiones y profundidad (Hernández et.al, 2011). Lo anterior apunta a que sería posible ir más allá de visualizaciones y recorridos en el proceso de enseñanza-aprendizaje, pero aún no surge una metodología validada para lograrlo.

\subsection{Problemática y Objetivo}

Experiencias previas desarrolladas en diferentes asignaturas del departamento de Arquitectura de la Universidad Técnica Federico Santa María avalan que el análisis de arquitectura promueve el desarrollo del pensamiento espacial (Marchesi, 1983) e histórico (Santiesteban, 2010), fortaleciendo, habilidades fundamentales para la formación de estudiantes de arquitectura. En este proceso, identificar el orden y la estructura profunda de los casos de estudio o 
propuestas de diseño resulta indispensable, pues permite comprender sus atributos y su totalidad arquitectónica (Norberg-Schulz, 1979; Clarke, Pause, 1997; Martí, 1993; Unwin, 1997).

Para la aproximación integral a las obras/diseños se trabaja con medios convencionales como fotografías, documentales, planimetrías y modelos físicos. Sin embargo, el efecto e impacto que la imagen en estos medios tiene sobre la comprensión del caso puede llegar a disociar la apariencia (i.e. figura) de la estructura profunda (i.e. forma) (Martí, 1993). En la práctica docente, hemos observado que la reintegración de las partes, posterior a la fragmentación inherente al proceso de análisis, que busca identificar componentes y atributos, resulta compleja con medios tradicionales de representación. Por tanto, a pesar de ser un proceso conducente al desarrollo de pensamiento espacial, en etapas iniciales ya se requiere de un nivel básico de esta competencia que puede no estar presente en el estudiante y que depende notablemente de experiencias espaciales previas. Es en este aspecto del proceso donde las tecnologías inmersivas, particularmente la RV, podrían operar como un medio amplificador de la capacidad de comprensión espacial de los estudiantes, entregando una experiencia que involucre la sensación de presencia en un entorno digital homologable al medio físico que alberga la obra o diseño, explicitando sus cualidades. No obstante, para lograr dicho cometido es necesario identificar una estrategia metodológica adecuada que garantice el aprendizaje.

Algunos experimentos previos -cinco workshops realizados en talleres presenciales, sumando un total de 88 estudiantes y 7 profesores- indican que la experiencia inmersiva facilita la integración de los diversos contenidos obtenidos previamente con medios tradicionales de representación (Fig. 1). Además, nos han permitido comprobar que, la experiencia virtual inmersiva e interactiva facilita la percepción y reconocimiento de cualidades espaciales con sus fortalezas y debilidades.

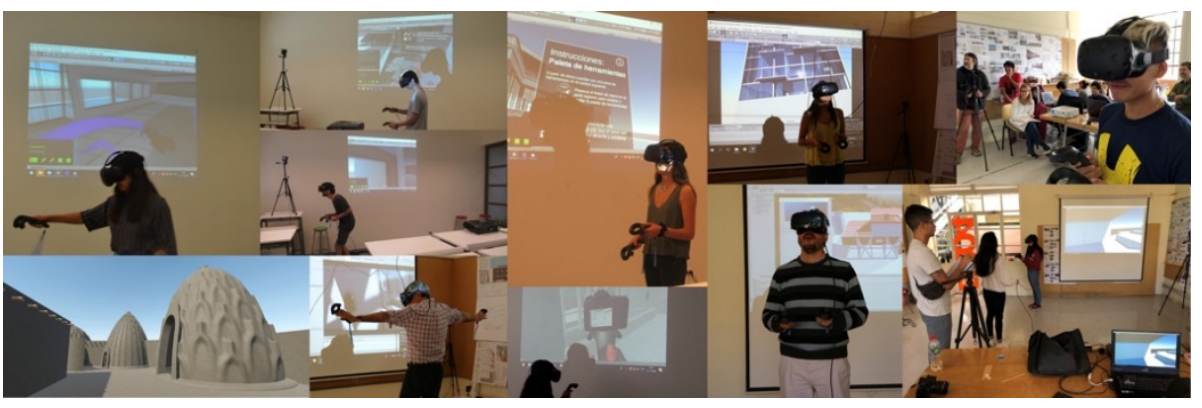

Figura 1. Experiencias de realidad virtual inmersiva realizadas con estudiantes y profesores entre 2018-2021 por los autores. Fuente: Elaboración propia, 2021. 
Por ello, las preguntas que motivaron esta investigación son: ¿Puede la RV contribuir efectivamente en el análisis y facilitar la comprensión de la obra? ¿Se puede lograr un acercamiento sistemático que garantice el aprendizaje mediante RV? Entonces, nos propusimos desarrollar y evaluar métodos e instrumentos que permitan estudiar crítica y comparativamente el impacto que la RV puede tener en el análisis arquitectónico.

\section{Metodología}

\subsection{Contextualización Metodológica}

Para cumplir el objetivo de la investigación nos propusimos indagar en las teorías de aprendizaje relacionadas con la experiencia. Identificar los contenidos fundamentales del análisis para comprender progresivamente el orden arquitectónico de las obras (Clarke, Pause, 1996), mediante el diseño de una experiencia de aprendizaje activo, basada en un instrumento de análisis arquitectónico interactivo. Implementar experiencias de evaluación para corroborar hipótesis con los usuarios objetivo (estudiantes de arquitectura) mediante una estrategia de análisis con grupo control, a fin de evaluar comparativamente el impacto de la Realidad Virtual Inmersiva (RVI) y la Realidad Virtual No Inmersiva (RVNI) en el proceso de enseñanza-aprendizaje.

El desarrollo estructural del instrumento se trabajó en función de la teoría de aprendizaje experiencial de David Kolb (1984), entendiendo este concepto, en términos sencillos, como el "aprender a través de la reflexión sobre el hacer" (Felicia, 2011). Esto permitió fundamentar la construcción de un "recorrido cognitivo" basado en cuatro momentos correlativos: Experiencia Concreta, Observación Reflexiva, Conceptualización Abstracta y Experimentación Activa. La decisión de usar dicha teoría se sustentó en el reconocimiento de similitudes y aproximaciones afines con el proceso de desarrollo proyectual implementado en talleres de arquitectura.

Los contenidos del instrumento desarrollado se configuraron mediante dos unidades de aprendizaje graduales, una de elementos fundamentales (Unidad 1) y otra de elementos variables (Unidad 2) (Unwin, 1997). La primera abordó cuatro temas que corresponden a definiciones y aplicaciones de "Elementos Fundamentales", "Espacios Fundamentales", "Organización y Relaciones Espaciales", y "Orden". La segunda unidad comprendió temas como "Luz", "Temperatura y Textura”, "Ventilación y Sonido", "Escala”, y “Tiempo-materia".

Cada tema de cada unidad se organizó según las cuatro instancias del aprendizaje experiencial mencionadas anteriormente. El recorrido secuencial 


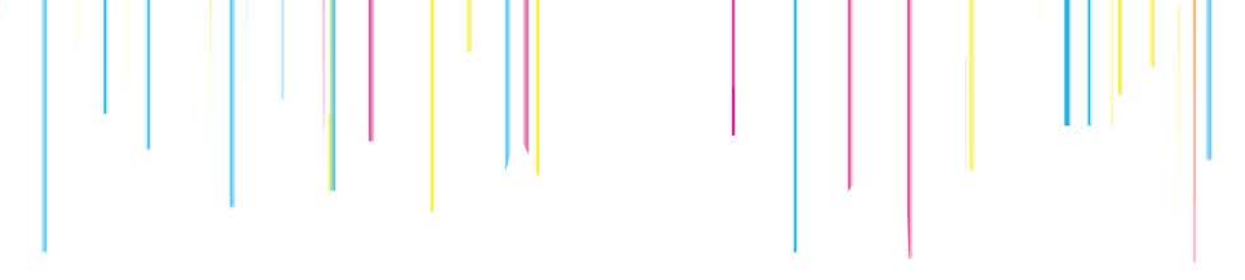

de unidades y temas contribuyó en la construcción paulatina de los resultados de aprendizaje (Bloom, 1956; Anderson \& Krathwohl, 2001). Por ello, se parte conociendo conceptos y definiciones fundamentales de la arquitectura para luego comprender su alcance, analizar sus variantes e invariantes $y$, finalmente, reconocerlos en una obra canónica.

\subsection{Métodos Detallados}

Una de las grandes dificultades a las que se ha enfrentado esta investigación, corresponde al contexto educativo actual asociado a la pandemia de COVID19 y el modelo de clases online. Por ello, el diseño se adaptó y la puesta a prueba del instrumento se desarrolló de manera remota.

Se realizó una convocatoria abierta a la comunidad de estudiantes, indicando los requerimientos tecnológicos necesarios para participar en las experiencias, presentándose 13 estudiantes con un rango similar de edades y avance curricular.

La puesta a prueba de este instrumento se trabajó con la Unidad 1, particularmente con 2 contenidos: "Elementos fundamentales de la Arquitectura" y "Espacios fundamentales de la Arquitectura".

Para evaluar comparativamente el impacto de la RV versus las herramientas tradicionalmente utilizadas (Ibrahim, Ramihian, 2010) ambos ciclos se aplicaron a tres grupos en paralelo. Éstos se organizaron en función de tres medios de representación (Fig. 2): Realidad Virtual Inmersiva (RVI) y Realidad Virtual No Inmersiva (RVNI), como grupos experimentales 1 y 2 respectivamente $\mathrm{y}$, para el grupo control, inicialmente referido a métodos tradicionales de representación (maquetas físicas), se utilizó una maqueta digital, observable sobre tablero con una herramienta de realidad aumentada (RA). Así se cumplió el objetivo fundamental de entregar una experiencia similar a la inspección de un modelo físico escalado, propio de los talleres de arquitectura.

Cabe destacar que el grupo de RVI trabajó con visores Cardboard entregados por el equipo. Si bien en las experiencias previas de evaluación la modalidad RVI se trabajó con equipos de alta gama (i.e. HTC VIVE), en esta ocasión fue necesario utilizar estos modelos, sujetos a los recursos tecnológicos disponibles por los usuarios, particularmente teléfonos inteligentes. 


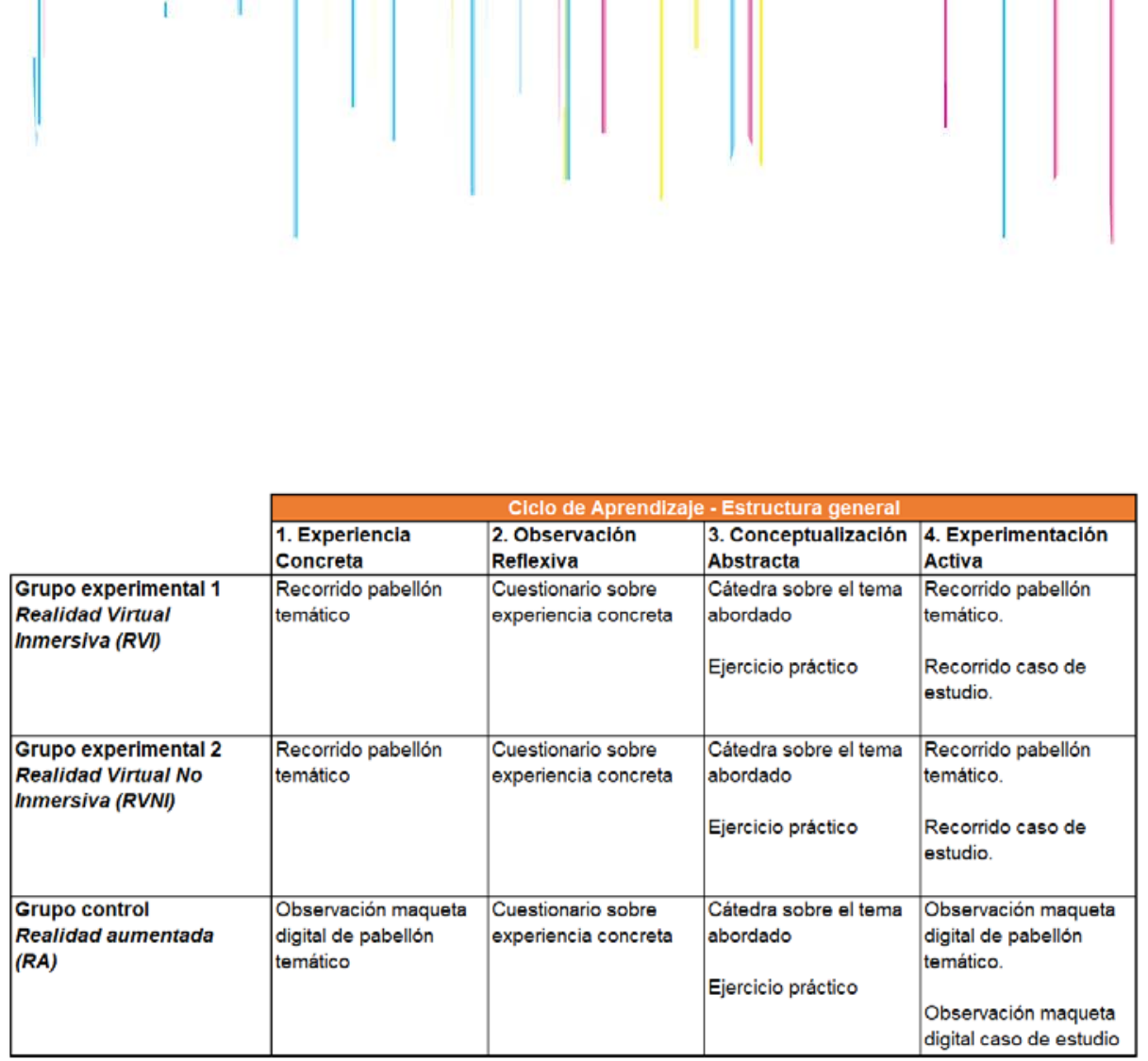

Figura 2. Diseño de experiencia, ciclo de aprendizaje general. Fuente: Elaboración propia, 2021.

\subsection{Desarrollo}

En un primer diseño de la experiencia interactiva, se planteó la implementación del software Kubity, una herramienta de visualización tridimensional que opera en computadoras de escritorio como aplicación web y, además, como aplicación de teléfonos inteligentes, con funciones de realidad virtual y aumentada, permitiendo la navegación de los modelos desarrollados (pabellones temáticos) y adquiridos (casos de estudio) para las experiencias (Fig. 3).

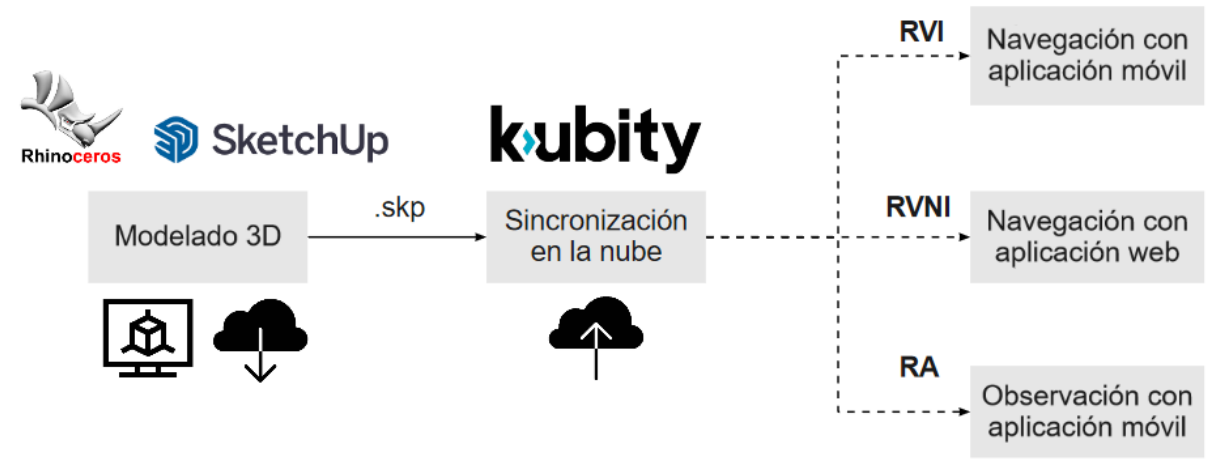

Figura 3. Esquema operativo desarrollo de experiencia de evaluación. Fuente: Elaboración propia, 2021.

La modalidad de RVI, operó con teléfonos inteligentes y visores Cardboard, instancia para la cual Kubity contribuyó con un modo de navegación sin 
botones basado en apunte y teletransporte, denominado Gaze \& Go, utilizando el giroscopio del dispositivo. Para RVNI se implementó la aplicación web del software, donde se utilizó ratón y teclado para la navegación, de modo similar a videojuegos de primera persona. En cuanto a RA, nuevamente se implementó la aplicación móvil que permitió detectar un plano de trabajo en el medio real, sobre el cual desplegar la maqueta digital, admitiendo su observación desde distintos ángulos con el movimiento y rotación del teléfono inteligente.

Todo el ciclo de aprendizaje (4 etapas) fue coordinado desde una sesión de videollamada con la aplicación Zoom. La Experiencia Concreta; primera etapa, consistió en la navegación u observación de un pabellón, donde se solicitó a los estudiantes identificar distintos elementos destacados y numerados en el mismo modelo, para lo cual se dispuso de formularios en línea. Esto implicó transitar desde una plataforma a otra (Fig. 4), condición inevitable bajo este modelo, puesto que el software no integra u admite un módulo que permita llevar a cabo el registro. Esa estructura se mantuvo en todos los casos en que se inspeccionó un modelo tridimensional.

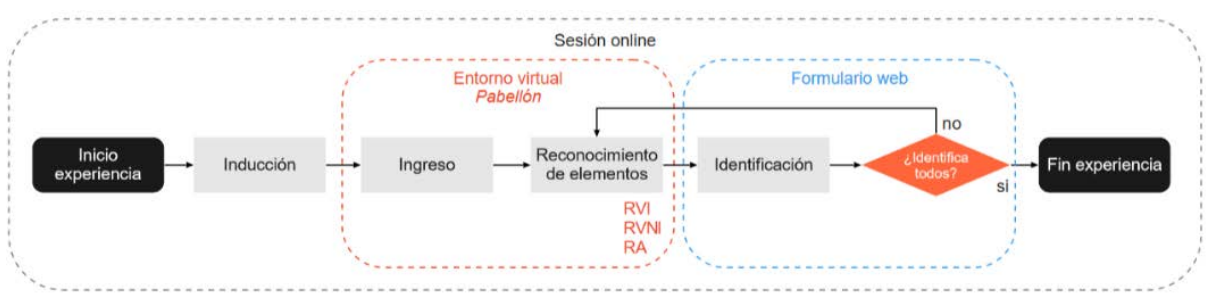

Figura 4. Diagrama de flujo experiencia navegación u observación de modelos 3D. Fuente: Elaboración propia, 2021.

Además, a los usuarios se les solicitó registrar, tanto su actividad en cámara mediante la videollamada, como el recorrido u observación realizado con computador o dispositivo móvil, según sea el caso (Fig. 5).

Luego de la Experiencia Concreta se implementó un cuestionario de las primeras impresiones respecto del pabellón visitado buscando registrar los conocimientos previos de los contenidos experimentados en lo que se denomina Observación Reflexiva. Posteriormente, se desarrolló la etapa de Conceptualización Abstracta realizando una cátedra de 20 minutos, donde se les explica cuál es el contenido del ciclo de aprendizaje, ya sea elementos o espacios fundamentales, entregando información recolectada de la literatura. Con esta actividad los estudiantes tuvieron la posibilidad de comparar sus conocimientos previos y evaluarlos mediante una experiencia con las descripciones convencionales de los elementos consultados, aplicando 


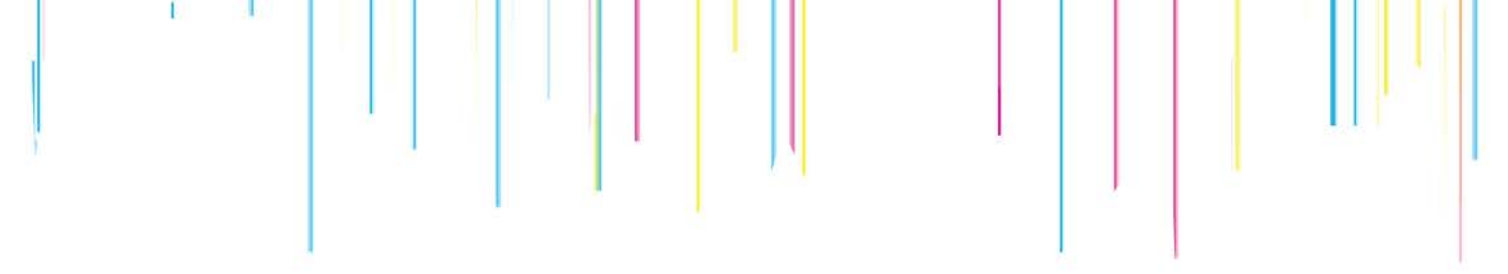

Estas ideas fueron reforzadas durante la entrevista grupal realizada al final de la experiencia, insistiéndose en implementar esta metodología para reforzar el proceso de enseñanza-aprendizaje de contenidos teóricos o facilitar el diseño arquitectónico. Esto refuerza el rol acelerador que la experiencia juega en el logro de los resultados de aprendizaje.

\subsection{Contenidos fundamentales e Instrumento de análisis arquitectónico interactivo}

La relevancia y pertinencia de la secuencia conceptual abordada en los recorridos para comprender progresivamente el orden arquitectónico de las obras también fue evaluada mediante encuesta en tres instancias de cada ciclo de aprendizaje. Una tras el primer recorrido por el pabellón respectivo elementos y espacios fundamentales-, otra después de la cátedra y al finalizar el ciclo. Los encuestados declararon que antes de la experiencia un 92\% conocía algunas definiciones y contenidos, y un $8 \%$ conocía todos los contenidos, pero no las definiciones. Sin embargo, cuando se les preguntó qué creían que mostraba cada pabellón, solo un $23 \%$ logró acertar en el primer ciclo, mientras que en el segundo solo acertó un 15\%. Tras el primer recorrido del pabellón de Elementos Fundamentales, un 46\% logró reconocerlos, mientras que, en el recorrido posterior a la cátedra, un 54\% obtuvo aciertos. Tras el primer recorrido del pabellón de Espacios Fundamentales, un 46\% consiguió identificarlos, mientras que en el recorrido posterior a la cátedra un $100 \%$ consiguió reconocerlos. Cabe destacar que en esta ocasión un 62\% identificó Elementos Fundamentales, reforzando los contenidos aprendidos en el primer ciclo. Otro momento evaluativo fueron las cátedras, donde se desarrolló un ejercicio de reconocimiento y descripción de Elementos, Espacios Fundamentales y Relaciones, mediante imágenes de proyectos, cuyos aciertos se detallan a continuación (Fig. 6).

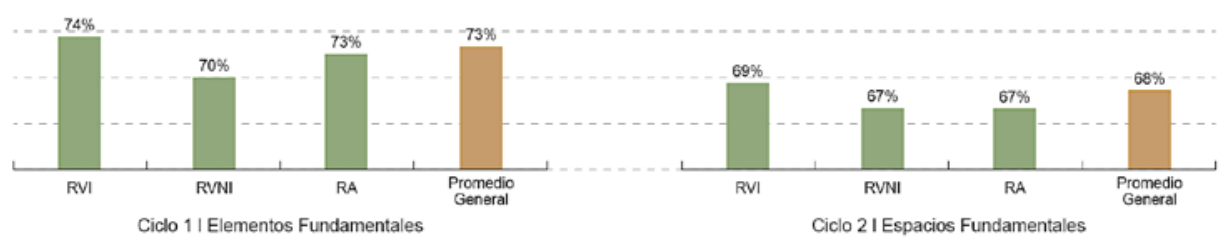

Figura 6. Porcentajes aciertos tras cátedras. Fuente: Elaboración propia, 2021.

En general, los contenidos lograron procesarse fácilmente durante la experiencia, el $100 \%$ de los participantes finalizaron ambos ciclos. Las mayores dificultades presentadas fueron por dificultades tecnológicas, como problemas en el desplazamiento o para encontrar la señalética asociada a componentes de análisis. Si bien los estudiantes conocen los términos, llama la atención que no tengan claras las definiciones ni logren reconocer elementos 
y espacios asertivamente, ya que el grupo se constituye principalmente por estudiantes de cursos superiores de la carrera. Por ello, se confirma que los contenidos definidos para ambos ciclos de aprendizaje requieren exponerse progresivamente para reforzar vacíos conceptuales, y el recorrido cognitivo potencia dicho reforzamiento. Entonces, la estructura metodológica del aprendizaje experiencial fortalece la dinámica expositiva progresiva y el refuerzo conceptual, garantizando el aprendizaje en materia de análisis. Por último, se destaca que los encuestados reconocen el dinamismo de esta aproximación, que fomenta nuevas estrategias de análisis arquitectónico y permite comprender la complejidad detrás de tal cometido.

\subsection{Comparación entre medios e impacto en el proceso de enseñanza- aprendizaje}

En cuanto al aporte que cada medio brinda a la experiencia, se revela que tanto RVI como RVNI permiten un acercamiento en primera persona a todos los espacios del modelo, favoreciendo la comprensión de contenidos. En contraste, la RA dificulta la observación de espacios interiores y detalles específicos al inspeccionar el modelo como si fuese una maqueta física, siendo su mayor potencial bordear el modelo y contemplar la organización ubicuamente. Por ello, para el análisis se requiere de planimetrías que permitan comprender mejor el caso de estudio. Se destaca que la RVI admite "sumergirse" en la experiencia, comprender claramente la diferencia entre elementos y espacios mediante la escala humana, y contemplar los detalles y la organización espacial general consecutivamente. En el caso de la RVNI se destaca que brinda una amplia perspectiva espacial de la totalidad y los elementos, permitiendo recorrer cómodamente los modelos y comprender los contenidos. Los participantes del grupo RA estiman que entrega gran libertad para rodear los modelos, relacionarlos con su entorno y apreciar la totalidad, considerándolo ideal para analizar el partido general de un proyecto u obra.

Entre las dificultades que presentaron los medios, se destaca que el $50 \%$ del grupo RVI manifestó incomodidad al momento de quitarse constantemente los visores Cardboard para llenar formularios, provocando mareos e incomodidad visual. Un $11 \%$ manifestó tener problemas constantes con su dispositivo móvil, lo que dificultó la experiencia. El grupo RVNI no manifestó problemas con la herramienta, pero un $30 \%$ evaluó críticamente la condición laberíntica de algunos pabellones y otro 30\% presentó problemas de conectividad durante la experiencia. Un $30 \%$ del grupo de RA tuvo problemas de incompatibilidad con el dispositivo móvil, retrasando el desarrollo de la experiencia sincrónica y un $60 \%$ indicó dificultad táctil para desplazarse o lograr llegar con precisión a ciertas zonas del modelo y seleccionar elementos.

Si bien esta experiencia inicial, no nos permite entregar resultados concluyentes acerca de una comparación de estas diferentes modalidades 


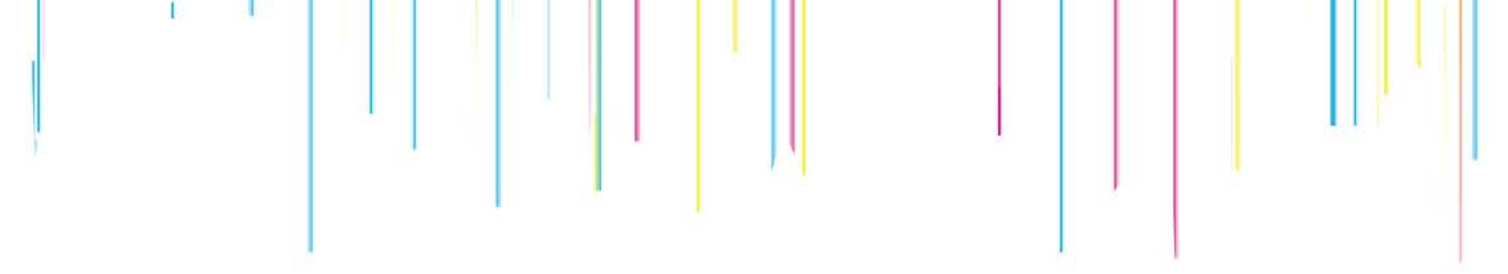

estudiadas debido al tamaño muestral, permite entender y confirmar la validación de la RVI como una herramienta de análisis frente a otros métodos de representación.

Finalmente, hay que destacar que el 92\% respondió el total de las encuestas y que del total de los participantes un 33\% evalúa la experiencia completa con nota 7 , un $42 \%$ con nota 6 y un $25 \%$ con nota 5 , en escala de 1 a 7 , cuyas justificaciones apuntan directamente a problemas técnicos y no de contenidos ni metodología. El 100\% de los participantes afirma que la experiencia ha sido valiosa y que definitivamente contribuye en el proceso de enseñanzaaprendizaje, datos que coinciden con las apreciaciones obtenidas en las experiencias previamente desarrolladas a esta medición.

\section{Discusión y conclusión}

El nuevo escenario educativo ocasionado por la crisis sanitaria de COVID-19 ha detonado la implementación de la virtualidad como una primera necesidad. La innovación en nuevas estrategias docentes que adopten esta virtualidad y que garanticen el aprendizaje efectivo, resulta indispensable. Tales estrategias requieren de recursos tecnológicos, lo que queda demostrado en este estudio, cuya disponibilidad resultó fundamental y donde un porcentaje sustantivo de usuarios no contaba con un sistema apto para RVI. Una encuesta desarrollada por el equipo para identificar la disponibilidad de recursos tecnológicos reveló que un alto porcentaje de estudiantes no sabe si cuenta con un teléfono inteligente con giroscopio. Por ello, se pone en duda la viabilidad de una integración del sistema RVI al modelo educacional curricular en curso, ya que algunos estudiantes podrían disfrutar de las virtudes de la inmersión mientras que otros no. Se concluye que un modelo de desarrollo con RVNI puede contribuir a una implementación horizontal y con mayor grado de confort y aceptabilidad, con resultados similares.

Si bien el estudio desarrollado aún depende de un muestreo mayor de usuarios, siendo un trabajo en curso, permite confirmar que la implementación de tecnologías de RV resulta eficaz en acelerar el entendimiento de obras y diseños arquitectónicos. Por lo mismo, se comprueba que la capacidad de navegar interiores de las obras inspeccionadas permite identificar rápidamente las cualidades de los elementos desde su relación con otros elementos próximos. Esto se diferencia de instancias con métodos de inspección desprovistos de puntos de vista sucesivos y que emulan un habitar en escala 1:1 (una "primera persona"), donde se requiere de una mayor capacidad de pensamiento espacial, usualmente nutrida por la acumulación de experiencias arquitectónicas y agilidad práctica en análisis para interpretar modelos a escala o planimetría. 
La metodología de aprendizaje experiencial es bien valorada por los participantes, reconociendo una estructura clara e identificando las virtudes de una exploración libre y reflexiva, exitosamente vinculada a una aproximación teórica y a una oportunidad de ajuste y puesta en práctica de los nuevos conocimientos adquiridos. Se comprueba que potencia la integración de herramientas docentes. Las observaciones de los participantes dejan en claro que los principales conflictos se sitúan en los aspectos tecnológicos, como la transición sucesiva entre plataformas, en este caso para navegación/observación y registro, incide negativamente en el confort; colocar y retirar las gafas de RVI constantemente produce mareos, así como retirar el foco de la cámara del teléfono inteligente al usar RA para responder sobre los elementos produce errores en el posicionamiento de la maqueta digital, conduciendo a desorientaciones. Por ello, la integración efectiva y eficiente de RVI y RVNI requiere evaluaciones de desempeño de los recursos tecnológicos.

A modo de trabajo futuro, se encuentra en desarrollo un software a medida, con el motor de videojuegos Unity, focalizado en requerimientos específicos de cada actividad, buscando la integración del recorrido, observación, identificación y registro en una misma plataforma (Fig. 7).

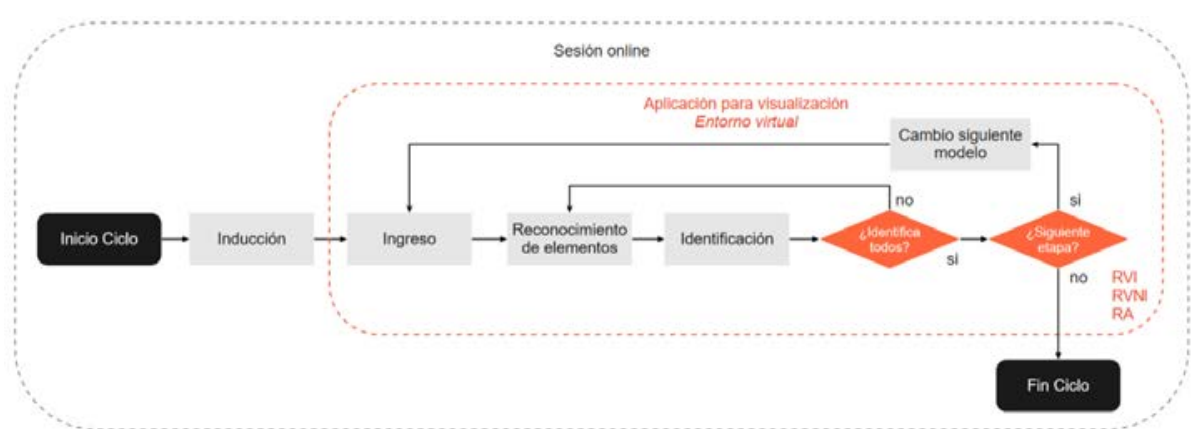

Figura 7. Diagrama de flujo experiencia navegación u observación de modelos 3D. Fuente: Elaboración propia, 2021.

En este desarrollo se prescinde de formularios externos y toda identificación de elemento o espacios se realiza desde la misma plataforma mediante paneles desplegables en el entorno virtual, enviando la información automáticamente a un formulario Excel, donde se recogen datos para el análisis y evaluación de indicadores. El diseño actual, aún considera que la catedra se desarrolle mediante la plataforma Zoom y se encuentra en evaluación la posibilidad de integrarla en la misma aplicación.

De este modo, pretendemos transformar este método y herramientas en una metodología de análisis arquitectónico interactivo. 


\section{Referencias}

Anderson, L.W. \& Krathwohl, D.R. (Eds.) (2001). A taxonomy for Learning, teaching, and assessing: A revision of Bloom's taxonomy of educational objectives. New York: Addison Wesley Longman.

Bloom, B. S. (1956). Taxonomy of educational objectives: The classification of educational goals; Handbook I: Cognitive domain. In M. D. Engelhart, E. J. Furst, W. H. Hill, \& D. R. Krathwohl (Eds.), Taxonomy of educational objectives: The classification of educational goals; Handbook I: Cognitive domain. New York: David McKay.

Clark, R. H., Pause, M., \& Castán, S. (1997). Arquitectura: temas de composición. Barcelona: Gustavo Gili.

Felicia, P. (Ed.) (2011). Handbook of research on improving learning and motivation through educational games: Multidisciplinary approaches. USA: iGi Global.

Ge, Y. (2019). Teaching Research on "Experience" Architecture History with VR Technology. Frontier of Higher Education, 1(1), 5-9. https://doi.org/10.36012/fhe.v1i1.569

Gutiérrez, D., \& Hernández, L. A. (2003). Potencial de la Realidad Virtual en el ámbito del patrimonio. Revista $\mathrm{PH}, 46,1-9$.

Hernández, L., Taibo, J., Seoane, A., \& Jaspe, A. (2011). La percepción del espacio en la visualización de arquitectura mediante realidad virtual inmersiva. EGA. Revista de expresión gráfica arquitectónica, 16(18), 252-261.

Hu, X., Ng, J., \& Lee, J. H. (2019). VR creation experience in cultural heritage education: A preliminary exploration. Proceedings of the Association for Information Science and Technology, 56(1), 422-426. https://doi.org/10.1002/pra2.00042

Ibrahim, R., \& Rahimian, F. P. (2010). Comparison of CAD and manual sketching tools for teaching architectural design. Automation in Construction, 19(8), 978-987.

https://doi.org/10.1016/j.autcon.2010.09.003

Kolb, D. A. (1984). Experiential learning: Experience as the source of learning and development. New Jersey: Prentice-Hall.

Kowalski, S., Samól, P., Szczepański, J., \& Dłubakowski, W. (2020). Teaching architectural history through virtual reality. World Transactions on Engineering and Technology Education, 18, 197-202.

Levis, D. (1997). Realidad virtual y educación. Recuperado de https://docplayer. es/332. 
Marchesi, A. (1983). Conceptos espaciales, mapas cognitivos y orientación en el espacio. Estudios de psicología, 4(14-15), 85-92. https://doi.org/10.1080/02109395.1983.10821355

Martí Arís, C. (1993). Las variaciones de la identidad. Ensayo sobre el tipo en arquitectura. Barcelona: Ediciones Del Serbal.

Navarro Delgado, I., \& Fonseca Escudero, D. (2017). Nuevas tecnologías de visualización para mejorar la representación de arquitectura en la educación. ACE: architecture, $\quad$ city, $\quad$ and $12(34), 238$. https://doi.org/10.5821/ace.12.34.5290

Norberg-Schulz, C., Avia, J. S., \& de Valderrama, F. G. F. (1979). Intenciones en arquitectura. Barcelona: Gustavo Gili.

Radianti, J., Majchrzak, T. A., Fromm, J., \& Wohlgenannt, I. (2020). A systematic review of immersive virtual reality applications for higher education: Design elements, lessons learned, and research agenda. Computers \& Education, 147. https://doi.org/103778. 10.1016/j.compedu.2019.103778

Santiesteban, A. (2010). La formación de competencias de pensamiento histórico. Clio \& asociados, (14), 34-56.

Sooai, A. G., Lake, R. C., Rafu, N. M., Mamulak, P. A. N., Meolbatak, E. M., Samane, I. P. A. N., ... \& Al-Azam, M. N. Developing Vernacular Architecture Learning Tools using 3D Virtual Reality. http://doi.org/10.13140/RG.2.2.34394.18888

Unwin, S. (1997). Analysing architecture. London: Routledge. 